\title{
A Rare Case of a Large Intracavitary Cardiac Thrombus
}

\author{
Jeffrey Brian Ziffra ${ }^{\mathrm{a}, \mathrm{b}}$, Gholam Zadeii ${ }^{\mathrm{a}}$
}

\begin{abstract}
Cardiac thrombi often present as a homogenous echodensity on cardiac imaging that may be incidentally discovered. A thrombus must be differentiated from vegetations, tumors and normal variant anatomy as their treatments are different. Cardiac thrombi are often located in cardiac chambers, most commonly the left atrium and left atrial appendage, where there is more stagnant blood flow. Thrombi may also form in structurally altered cardiac chambers as well as seen in dilated cardiomyopathy and in ventricular aneurysms. Symptoms, if present, may vary from dyspnea, angina and compressive symptoms. Currently, transthoracic echocardiography, transesophageal echocardiography and magnetic resonance imaging (MRI) are the accepted modalities to evaluate cardiac masses. MRI has been used with more frequency due to its ability to delineate mass borders, determine endocardial involvement and in its ability to detect blood flow. Few cases have been described of a large cardiac thrombus extending through the valves to include multiple chambers. In our case presentation, we describe a 91-year-old female with no significant cardiac history who presented with a non-ST elevation myocardial infarction where a transthoracic echocardiogram revealed a large intracavitary thrombus.
\end{abstract}

Keywords: Cardiology; Thrombus; Echocardiography

\section{Introduction}

Large cardiac thrombi traversing across cardiac valves have rarely been reported. Cardiac thrombi often occur when conditions predispose to blood flow stagnation and are often found in the left atrium and appendage. The differential diagnosis of a cardiac mass includes thrombi, tumors, vegetations and variants of normal structures. The treatments of each of these are quite different. It is prudent that clinicians know what modalities to use to differentiate cardiac masses.

Manuscript accepted for publication June 08, 2016

aDepartment of Cardiology, Mercy Medical Center, North Iowa, Mason City, IA, USA

${ }^{b}$ Corresponding Author: Jeffrey Brian Ziffra, Department of Cardiology, Mercy Medical Center, 1000 4th St., Mason City, IA 50401, USA.

Email: jeffziffra@gmail.com

doi: http://dx.doi.org/10.14740/jmc2553w

\section{Case Report}

The patient was a 91-year-old female with a past medical history of hypertension, dyslipidemia and dementia who presented to the emergency room from her assisted living facility for vomiting, weakness and non-specific symptoms. The patient presented several days after she was discharged from the emergency room for one episode of coffee round emesis. During her prior visit, her vital signs and laboratory values were stable and her symptoms were under control allowing her to return to her assisted living facility. She then had a recurrence of vomiting, this time non-bilious and non-bloody in nature, which prompted her to be admitted to the emergency room at an outside hospital. Upon evaluation, she denied any angina, dyspnea or syncope.

Initially, her physical exam was significant for a temperature of $98.0^{\circ} \mathrm{F}$, blood pressure of $117 / 49 \mathrm{~mm} \mathrm{Hg}$, pulse rate of 77 beats per minute and respiratory rate of 22 respirations per minute, and she was saturating $100 \%$ on $2 \mathrm{~L}$ nasal cannula. The patient was alert, aware, oriented and not in acute distress. Her head was atraumatic and normocephalic. Her oral mucosa was moist and without lesions. Her neck was supple, no carotid bruits were appreciated and her neck veins were flat. Her heart was regular rate and rhythm and no murmur was appreciated. Her lungs were clear to auscultation bilaterally, and there was no wheezing or rales appreciated. Her abdomen was soft and bowel sounds were present. There was no lower extremity edema appreciated.

Her initial laboratory data were significant for a sodium level of 137, potassium level of 4.5, chloride level of $108, \mathrm{CO}_{2}$ level of 25 , BUN level of 33, creatinine of 1.69 , white blood cell count of 19.64, hemoglobin of 9.8, hematocrit of 29.9, platelets of 116, alkaline phosphatase level of 55, aspartate aminotransferase level of 16, alanine aminotransferase level of 19, and troponin I level of 2.95. The patient was also found to have hemoccult positive stool. A chest X-ray was performed which revealed cardiomegaly, normal pulmonary vasculature and a large $14.3 \mathrm{~cm}$ hiatal hernia. Her initial EKG demonstrated normal sinus rhythm with a heart rate of 77 beats per minute, without any ST-T wave changes. Because of her elevated troponin level, she was transferred to our institution to be evaluated by the cardiology service for further management of her non-ST elevation myocardial infarction.

The patient was started on medications for acute coronary syndrome given her elevated troponins. Her emesis was treated as an anginal equivalent as well. She received a $325 \mathrm{mg}$ 


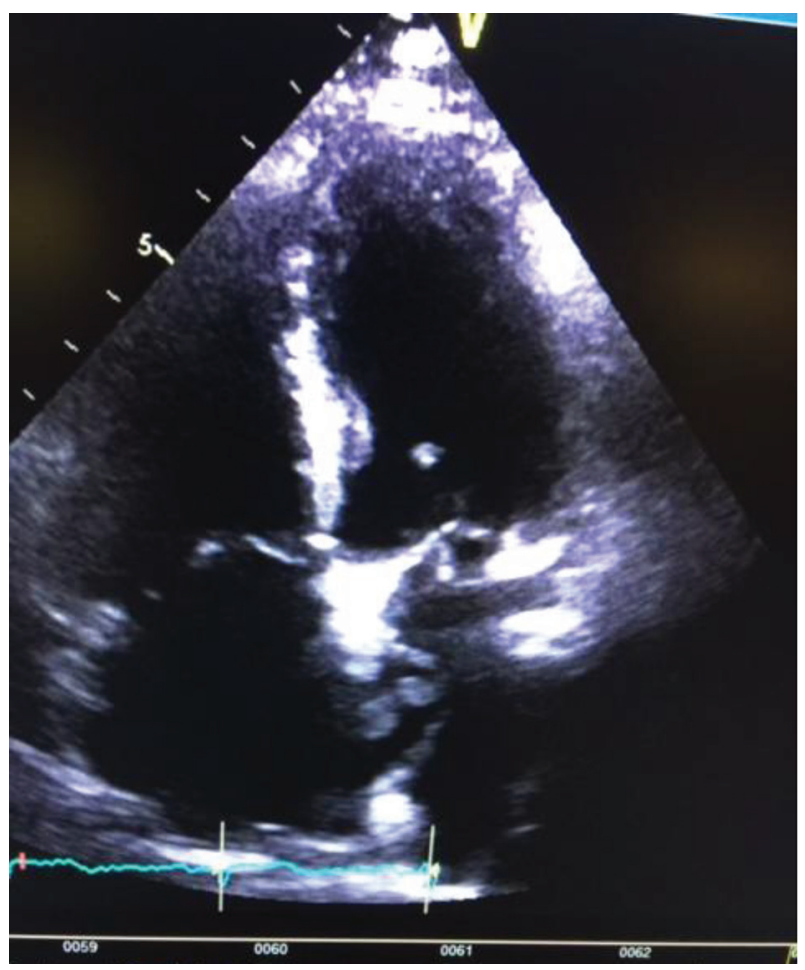

Figure 1. Apical four-chamber view showing thrombus hitting the intraatrial septum and crossing the mitral valve towards the apex of the left ventricle.

aspirin orally and was started on metoprolol tartrate $12.5 \mathrm{mg}$ three times a day. A transthoracic echocardiogram (TTE) was ordered to evaluate her left ventricular function and her wall motion. The TTE revealed a low-normal ejection fraction of $50 \%$ with septal wall motion hypokinesis. Enlargement of the left atrium, right atrium and right ventricle was demonstrated. Her right ventricular systolic pressure was severely elevated at $93 \mathrm{~mm} \mathrm{Hg}$. A large, mobile thrombus was noted in the right atrium extending to the right ventricle. The thrombus was also noted in the left atrium and left ventricle extending across the aortic valve. There was also moderate to severe tricuspid regurgitation noted by Doppler echocardiography (Figs. 1-3). There were no previous echocardiograms at our institution for comparison.

The patient was treated as non ST-elevation myocardial version type one versus non ST-elevation myocardial infarction type two secondary to demand ischemia from sepsis. Along with the oral aspirin and beta-blocker, the patient was then started on anticoagulation with heparin and coumadin. The patient was also requiring anticoagulation as she had developed atrial fibrillation during this hospitalization with risk factors placing her at higher risk for thrombus formation. The patient did not have a history of documented atrial fibrillation in the past. She was started on antibiotics for suspected aspiration pneumonia given her leukocytosis in the context of vomiting. Her renal function and leukocytosis continued to improve throughout her hospital stay. Because of the very large thrombus, surgery was considered but she was ultimately deemed not to be a surgical candidate. Angiogram was also considered

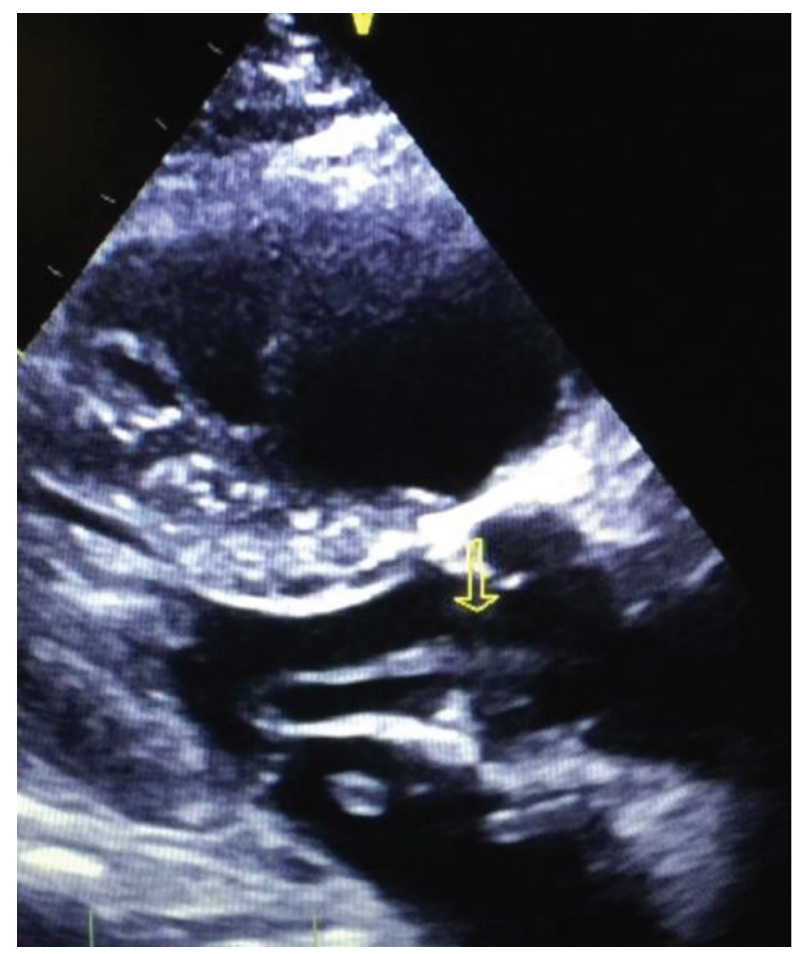

Figure 2. Parasternal long axis view of an intracavitary thrombus seen in the left atrium and in the left ventricle crossing the aortic valve into the aorta.

for evaluation of her coronary anatomy for which the patient declined opting for medical management. She continued her coumadin for anticoagulation until she obtained therapeutic international normalized ratio (INR) levels. The patient was then ultimately discharged back to her care facility.

\section{Discussion}

A cardiac thrombus is part of the differential diagnosis of a discovered cardiac mass. Clinicians must have a strong index of suspicion to search for a cardiac mass as the symptoms are quite variable if present and are also non-specific. Because the treatment modalities differ, a cardiac mass must be further differentiated if discovered. The incidence of cardiac thrombus has not previously been reported other than dated autopsy studies including Garvin who described a $34.4 \%$ incidence of intracardiac thrombi in consecutive autopsied patients who had died secondary to heart disease. Other similar studies have reported a wide variation of incidences of thrombi specifically in the left ventricle with lower incidences described in the right ventricle and right atrium [1]. For most cases, thrombi are best described in the left atrium as this is a location of blood flow stagnation. The atrium chambers are under a lower amount of hemodynamic pressure and velocity compared to ventricles making these chambers more thrombogenic. The left atrial appendage, especially if the patient is in atrial fibrillation, is also a very common area for a thrombus to develop. If the patient exhibits any symptoms, they can vary from dyspnea to ob- 


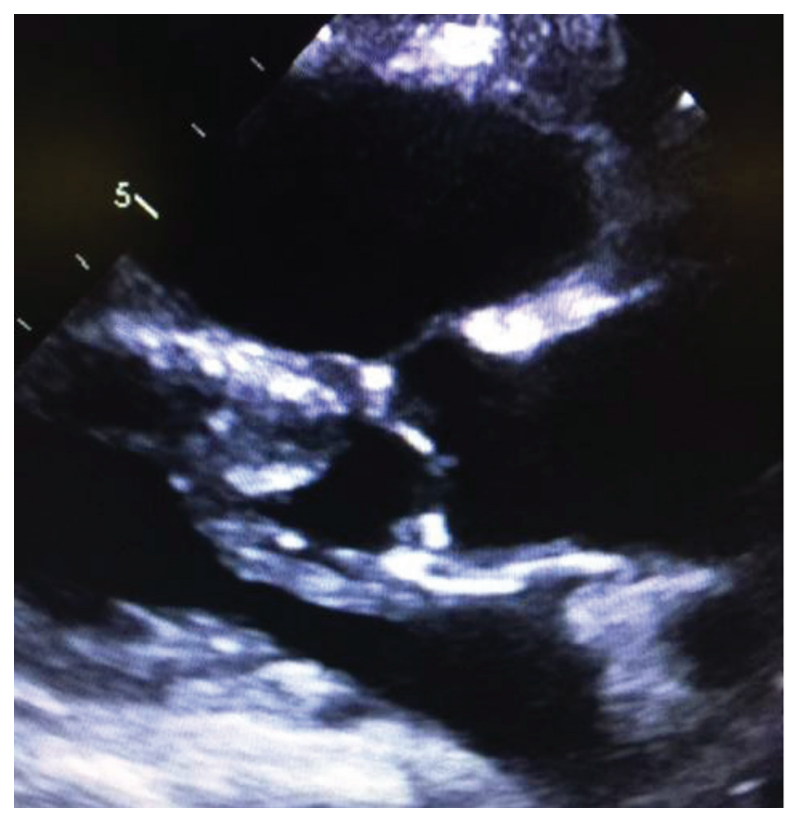

Figure 3. Intracavitary thrombus attached to the anterior wall of the left atrium and including the left ventricle as well.

structive symptoms. Thrombi described outside the left atrium are more often found in patients with dilated cardiomyopathy and ventricular aneurysms. Due to the altered architecture in dilated and aneurysmal ventricles, they will endure lower velocities and pressures than normal chambers [2].

The differential diagnosis for a cardiac mass includes tumor, vegetation, thrombus and normal anatomical structures. TTE is portable, cheap and non-invasive, making it the preferred first screening test for a cardiac mass. Contrast can be added to delineate borders and assist in further classification. Echocardiography has many limitations in differentiating types of cardiac masses however. Several studies have described TTE as a modality to evaluate a cardiac thrombus with an overall decreased specificity and sensitivity especially in comparison to magnetic resonance imaging (MRI). M-mode can sometimes add increased utility to echocardiography in detection of a thrombus; however, it is not very specific [3-5]. Transesophageal echocardiograms can provide greater detail and are particularly useful for locating a vegetation. Compared to transthoracic echocardiography, transesophageal echocardiography has an increased specificity and sensitivity but is more invasive.

MRI provides advantages over echocardiography in distinguishing between thrombi, vegetations, tumors and anatomical structures. It offers increased sensitivity and specificity in the differentiation of a cardiac mass. Compared to echocardiography, MRI provides greater delineation of mass borders and can provide greater soft tissue characterization. MRI can more accurately detect size, degree of involvement, vascularity, lesion mobility, fat content, and the presence of central necrosis due to its high spatial and temporal resolution. Locating a vascular supply is very important specifically in detecting tumors. MRI has been extensively studied in its ability to distinguish between types of cardiac tumors. The addition of gadolinium can provide further enhancement and more detail [6]. Once a cardiac mass is detected, MRI is a great tool to help provide further characterization and is less invasive than surgery or biopsy.

Few cases have been described of large intracavitary masses. Starkey described a case of a cardiac thrombus which travelled across the tricuspid valve lying in the right atrium and right ventricle which was detected by echocardiogram. The patient received thrombolytics with IV streptokinase for this large thrombus. The patient eventually succumbed to a massive pulmonary embolism. They further describe that after investigating 2,000 autopsies, four cases of right ventricle embolism were reported as the cause of death, making it quite rare [3]. Lee described a case of a 49-year-old woman with symptoms consistent with fluid overload. During diagnostic testing, she was found to have hyperechoic masses across the left ventricular apex and mitral annulus with a heterogeneous mass at the right atrium. They then used weighted MRI to help differentiate between cardiac masses, as they suspected the presence of multiple malignancies. After further differentiation, two of these masses were found to be thrombi and the latter was found to be a myxoma. After this discovery, heparin was started helping to dissolve two of the three masses. The tumor was excised and the patient made a full recovery [7].

We describe a case of an incidentally discovered intracavitary mass extending from the left atrium, across the mitral valve to the left ventricle and across the aortic valve. A thrombus was also visualized in the right atrium and right ventricle although it was unclear whether this was all one contiguous clot. In our literature review, we have yet to see such a thrombus described. As noted, right-sided thrombi have been reported to have a poor prognosis especially in the setting of hemodynamic compromise or right ventricular dysfunction [8]. The patient did not have any risk factors for thrombi such as systolic dysfunction, ischemic or dilated cardiomyopathy, prosthetic valves, ventricular aneurysms or atrial fibrillation on presentation. She subsequently later developed atrial fibrillation during her hospitalization. The patient ultimately decided against surgery and was placed on anticoagulation prior to discharge. Clinicians should be aware of conditions that may predispose patients to large intracavitary thrombi and be aware of the poor prognosis. If a cardiac mass is found incidentally, contrast can be added to echocardiography or a more specific modality such as MRI can be used to further differentiate the structure. MRI can add contrast sequences to detect blood flow, which would not be present in the case of a cardiac thrombus.

\section{Grant}

No grants were awarded to the authors.

\section{Conflicts of Interest}

We have no competing interests. We have not had any funding for this case report. We have followed standards for ethical approval and guarantor. We have both adequately contributed to 
the authorship of this manuscript and have no other acknowledgements.

\section{References}

1. Waller BF, Rohr TM, McLaughlin T, Grider L, Taliercio CP, Fetters J. Intracardiac thrombi: frequency, location, etiology, and complications: a morphologic review - Part II. Clin Cardiol. 1995;18(9):530-534.

2. Mann DL, Zipes DP, Libby P, Bonow RO. Braunwald's Heart Disease: A Textbook of Cardiovascular Medicine, Single Volume, 10th Edition.

3. Starkey IR, de Bono DP. Echocardiographic identification of right-sided cardiac intracavitary thromboembolus in massive pulmonary embolism. Circulation. 1982;66(6):1322-1325.

4. Srichai MB, Junor C, Rodriguez LL, Stillman AE, Grimm RA, Lieber ML, Weaver JA, et al. Clinical, imaging, and pathological characteristics of left ventricular thrombus: a comparison of contrast-enhanced magnetic resonance imaging, transthoracic echocardiography, and transesophageal echocardiography with surgical or pathological validation. Am Heart J. 2006;152(1):75-84.

5. Stratton JR, Lighty GW, Jr., Pearlman AS, Ritchie JL. Detection of left ventricular thrombus by two-dimensional echocardiography: sensitivity, specificity, and causes of uncertainty. Circulation. 1982;66(1):156-166.

6. Luna A, Ribes R, Caro P, Vida J, Erasmus JJ. Evaluation of cardiac tumors with magnetic resonance imaging. Eur Radiol. 2005;15(7):1446-1455.

7. Lee WC, Huang MP, Fu M. Multiple intracardiac masses: myxoma, thrombus or metastasis: a case report. J Med Case Rep. 2015;9:179.

8. Koc M, Kostrubiec M, Elikowski W, Meneveau N, Lankeit M, Grifoni S, Kuch-Wocial A, et al. Outcome of patients with right heart thrombi: the Right Heart Thrombi European Registry. Eur Respir J. 2016;47(3):869-875. 